\title{
Automatic charging of an energy harvesting powered sensor node from controllable energy source
}

\author{
Juha Petäjäjärvi, Jarkko Kaleva, \\ Konstantin Mikhaylov \\ Centre for Wireless Communications, \\ University of Oulu, Finland \\ \{firstname.lastname\}@oulu.fi
}

\author{
Heikki Pulkkinen, Jukka Ahola, \\ Max Björkgren \\ Helvar Oy Ab \\ Espoo, Finland \\ \{firstname.lastname\}@helvar.com
}

\begin{abstract}
It is expected that the number of the Internet of Things (IoT) devices will grow significantly in the coming years. Batteries, which are today the most common source of energy supply for these devices, have many limitations. The limited capacity and the negative environmental effect of their production and recycling are among the most crucial ones. Energy harvesting has been proposed to overcome this problem. Over the past decade, the academia has produced thousands of papers on this topic, but it is still difficult to find a commercial sensor node operating solely with energy harvesting. One of the major reasons for this is the highly opportunistic and unreliable nature of the energy income from most of the available ambient energy sources. In this paper, we address this problem by proposing a control loop enabling an energy harvesting sensor to request a recharge whenever needed. Energy-wise, the proposed method effectively transforms the operation mode of a passive wireless device from opportunistic to proactive, thus enabling an application to operate in a more predictable manner. The feasibility and efficiency of the proposed approach is demonstrated by implementing a real-life prototype Bluetooth Low Energy capable sensor node equipped with photovoltaic energy harvester, which can communicate and request a recharge from a commercial indoor smart lighting system.
\end{abstract}

Keywords-Bluetooth Low Energy; energy harvesting; IoT R\&D platform; smart light; quality of service

\section{INTRODUCTION}

Wireless sensor networks (WSN) are often treated as one of the key enablers of the upcoming fifth generation $(5 \mathrm{G})$ mobile networks and IoT. The range of IoT applications is vast: from smart home to industry, and from traffic monitoring to healthcare, if to name just a few. It is predicted that the number of sensor nodes will increase substantially in the next couple of years [1-2].

The applications implying sensor's mobility or deployment of sensor nodes in the places where it is expensive or difficult to implement wiring, are today mostly powered with one or multiple batteries. The energy coming from the batteries is used to power the sensor node's microcontroller, radio transceiver, sensors and actuators that enable it to carry out the assigned tasks. Even though the commercial sensor nodes today are rather energy efficient and can feature a lifetime of several years, the batteries still need to be changed or recharged at some point, which might create substantial spikes to the operational, namely maintenance, expenditures. One should not underestimate also the negative environmental impact both for production of a battery and recycling [3].

These considerations provide just one motivation for considering enablement of batteryless IoT applications. Another motivation comes from the fact that the current state of technology made many of the sensor node's operations such as wireless communication, signal processing, measuring of many physical parameters, and especially sleep mode, decently low energy consuming. This has substantially reduced the average consumption of a contemporary sensor node, bringing it closer to the levels, which can be compensated with the tiny amounts of the ambient energy collected by a sensor node from its environment.

There are several possibilities for harvesting the energy from the environment, e.g., light, vibration, temperature difference, electromagnetic field, and ambient RF transmissions. The availability and the amount of the energy, which can be collected from many of these sources, highly depends on the environment. To give an example, a sensor node equipped with a piezo-based vibration harvester may collect decent amount of energy for its operation when attached to an industrial machine but may never get enough energy to send a single radio frame if installed on a wall of an office. This illustrate the importance of the correctly matching the energy harvesting method and the environment of operation.

When this comes to the niche of environment monitoring IoT applications, light often becomes the most perspective option for getting the energy. Sunlight contains such a large amount of energy that even with a small photovoltaic panel the sensor node can harvest the needed energy. When the sun is down, artificial lighting can be used to enable continuous operation for many IoT devices.

Energy harvesting from light has been utilized for long time already, but it continues to evolve. The maximum efficiency of a photovoltaic cell at the moment is $46 \%$ while it was around $40 \%$ a decade ago [4]. Indoors, because the artificial lighting produces much narrower bandwidth than the sun, the achieved efficiency is much lower. The harvested power per square centimeter in outdoors and in indoors is in the order of $10 \mathrm{~mW}$ and $0.01 \mathrm{~mW}$, respectively [5]. 
Such high variation of the harvested energy, together with the possibility of no light being present at all, e.g., during the nighttime when the lighting is turned off, makes operation of typical light-powered sensor node highly opportunistic. This limits the use of such solutions both in outdoors and indoors. Meanwhile, the latter ones feature both the already deployed widespread artificial lighting deployments and have the need for efficient large real estate monitoring solutions that are an important part of facility management digitalization.

Therefore, in this paper we introduce one possible solution to this problem by enabling a control loop by using a sensor node, which can request a power source, such as a smart light, to switch on thus enabling the sensor node to replenish its energy. As a result, the proposed method enables to improve the reliability of the system's operation and guarantee certain quality of service for the target application.

The rest of the paper is organized as follows. Related work is covered briefly in Section II. Section III introduces the concept of controlling an energy source with a resource constrained sensor node. An experimental setup of the concept detailed in Section IV, which is followed by performance results of the sensor node in Section V. Section VI presents the conclusions.

\section{RELATED WORK}

Wireless sensor nodes powered with energy harvesting is certainly a hot topic, which can be seen from the number of research papers. Just in the IEEE digital library, there are tens of surveys alone of the topic. Generic surveys typically focus on different harvesting techniques, performance and challenges (e.g., [6-9]), but there are also surveys with a narrower focus such as wireless power transfer (WPT) [10-12], and media access control (MAC) protocols for energy harvesting [13].

The real-life prototypes of the light-powered WSN nodes have been reported, e.g., in [14]. When this comes to other sources, the WPT solutions were reported, e.g., in [15] and the piezo-based solutions were introduced in [16]. The development kits, composing an energy harvester and a sensor node are available, e.g., from Cypress [17].

Therefore, the lack of the commercial off-the-shelf WSN nodes operating solely with energy harvesting is rather surprising. One of the major reasons for this, as discussed, e.g., in [7], is that the harvested energy is often not available all the time and thus such sensors cannot guarantee decent level of reliability. This fact has raised the interest towards the methods based on dedicated WPT, which are more predictable. The downsides of this approach are the need for special infrastructure composed of the wireless power transmitters and the low efficiency of omnidirectional wireless power transfer.

Nonetheless, to the best of our belief, the problem of improving the reliability of operation of energy-harvesting powered sensors can be also addressed by instrumenting a control loop, giving a sensor some mean to control its potential energy sources located in its proximity.

\section{SYSTEM CONCEPT}

Consider a WSN node equipped with one or multiple energy harvesters and located in an environment, where the potential energy sources of the sensor node can be controlled. The most straightforward example, which we will use to illustrate the concept, is composed of a sensor node equipped with a photovoltaic panel, which is located under a smart lighting system. Another possible alternatives can be, e.g., a WPT harvester near to respective transmitters, or even a vibration harvester in an environment with controllable vibration.

An example system concept of the proposed controllable energy source for powering a sensor node is illustrated in Fig. 1. The system includes four components, namely the sensor node, a controllable light source (e.g., a lamp), a light controller (e.g., capable of switching the light on/off or adjusting the light's intensity), which is connected to a cloud via a gateway. The wireless link could be implemented with many different radio technologies as long as they consume low power while transmitting and receiving (e.g., ZigBee or Bluetooth Low Energy (BLE)). This ensures longer lifetime for the sensor node.

The general logic of the system's operation is as follows. While the energy income from the photovoltaic panel is sufficient, it continues execution of its application. Once the energy income gets low (e.g., during the weekend or evening times, when the lights go off) and the amount of energy available in the buffer falls below a certain threshold, the passive wireless sensor node establishes a wireless connection with the light controller and requests switching the light on. The latter processes the request and may switch the illuminator on. Once passive wireless device replenishes the energy to certain level, it notifies the light controller, which will switch the illuminator off.

The proposed system provides a mechanism for sustainable operation of the photovoltaic energy harvesting enabled passive sensor node by providing them a mechanisms to request by the means of wireless communication switch on or off the nearby lights. The system can be used indoors and outdoors in the context of different applications, such as various distributed measurement and control systems (e.g., indoor heating, ventilation and air conditioning (HVAC) system, outdoor traffic control systems, and infrastructure monitoring system).

Obviously, the proposed concept has several notable open issues, in respect to its implementation dealing with the security, scalability and efficiency. First, both the sensor and the source of light require possessing the same wireless communication interface. Second, the wireless communication needs to be secure and reliable, and also energy efficient. Third, the solution needs to be scalable - both in respect to the increase of the number of the sensor nodes and the number of energy sources (e.g., lamps). This may demand availability of efficient sensor node localization solutions on one hand and mechanisms of controlling individual energy sources on the other. Due to the practical constraints, in what follows we address only the implementation of a single-sensor single 


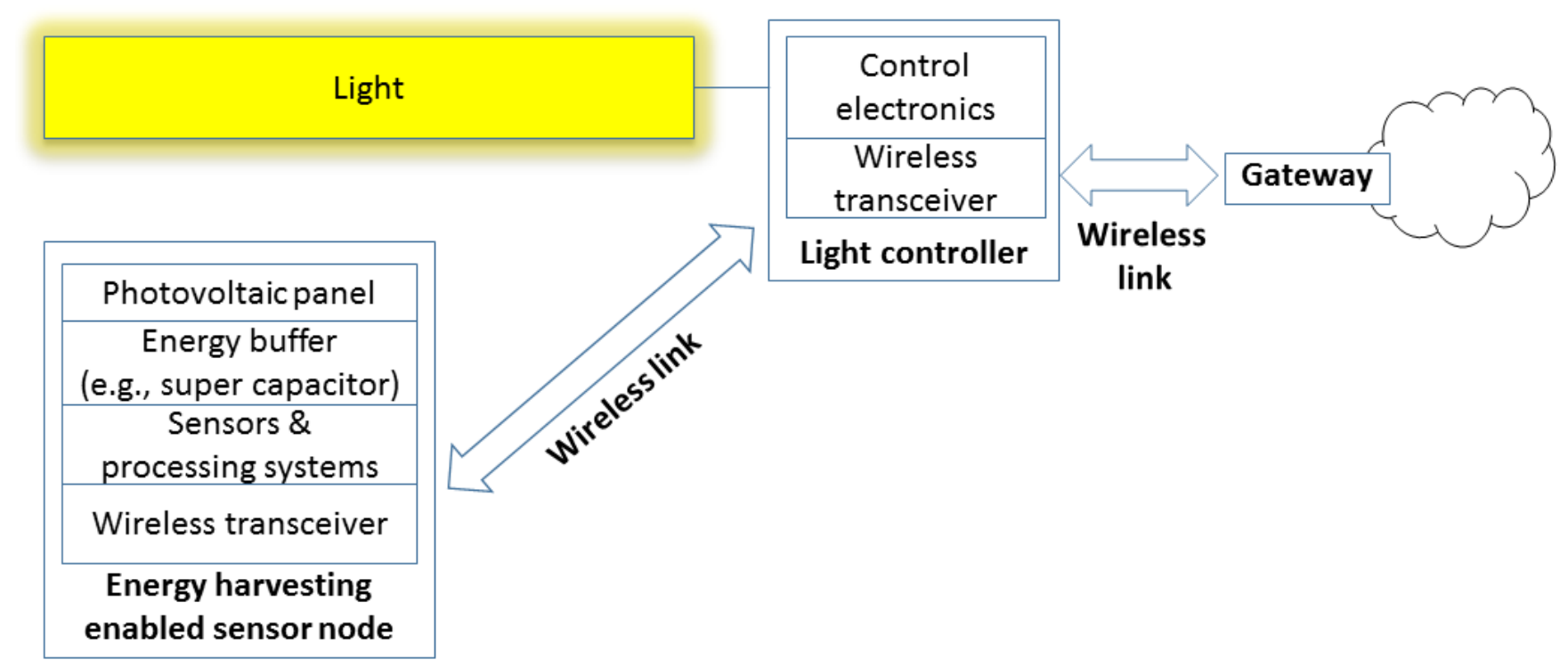

Fig. 1. A structure diagram of the illustrative pro-active energy harvesting system.

source case, leaving the more sophisticated scenarios for further studies.

\section{IMPLEMENTATION}

The implementation of the introduced in Fig. 1 system's proof-of-concept solution was carried out collaboratively by Centre for Wireless Communications (CWC) and Helvar Oy $\mathrm{Ab}$. The sensor node has been designed and instrumented by CWC, while the intelligent lighting solution has been provided by Helvar.

\section{A. Modular sensor node}

The sensor node is based on modular IoT research platform developed at CWC [18]. By stacking together different hardware modules, the platform provides the desired functionalities. The module options at the moment include various power sources, processing units, wired and wireless transceivers, sensors, and actuators. Once the sensor node is built, it automatically discovers and identifies all the connected modules and obtains the suitable software.

The passive sensor node engineered for this study is depicted in Fig. 2. It includes the main board composing the special buck-boost DC-DC converter circuitry (built around TI's BQ25570 chipset) with $1 \mathrm{mF}$ capacitor-based energy buffer and TI's multi-standard CC2650 system on chip (SoC), which can communicate over BLE and ZigBee. On top of the main board are attached a sensor module with six sensors, and a power module suitable for harvesting energy from light.

In order to maximize the lifetime for the sensor node, it was programmed to stay in low power sleep mode most of the time. Periodically the sensor node woke up, made a measurement, transmitted data to the smart lamp, and went back to sleep mode.

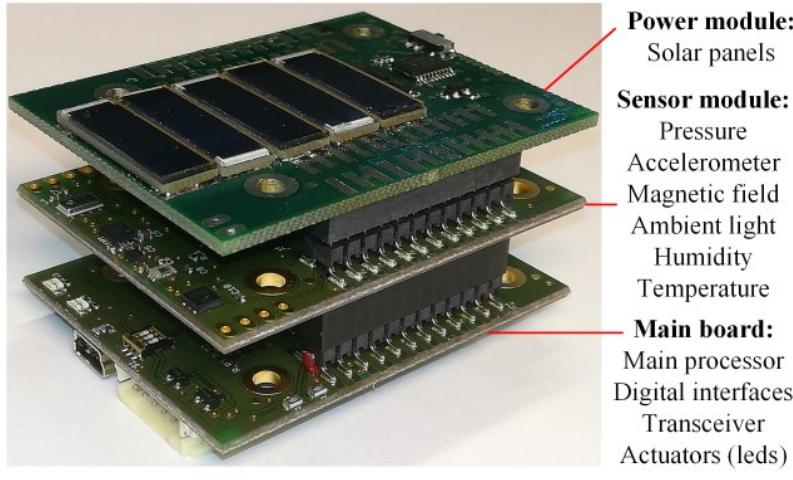

Fig. 2. Modular sensor platform used in the implementation consisted of solar panel and sensor module installed on top of the main board.

\section{B. Smart light system}

The smart light solution is provided by Helvar. Each lamp is equipped with a passive infrared sensor (PIR) and communicates with a gateway through a mesh BLE connection. The gateway is connected to the Helvar's cloud system that gathers information, e.g, about PIR activations. The smart lamp can be also controlled from the cloud.

\section{Communications}

The wireless communications was implemented using BLE. The BLE v5.0 standard has been already available since December 2016, but due to the lack of commercial chips at the time of implementation phase, version 4.2 was used. The main reason for choosing BLE is that it is rather simple and operates with rather high over-the-air data rates, both of which positively affect the energy consumption. 
In order to minimize the cumulative consumed power for communications, the sensor node and the light controller do not establish a connection, but communicate instead using the modified broadcast advertisement messages. The sensor node periodically transmitted measurement data of the environment and the amount of charge value. When the sensor node notices that it is running out of power, it transmits a request to turn the lights on. Alternatively, this could have been implemented in a way that the smart lamp learns when the sensor node needs recharging and could automatically switch on. Downlink communications was not utilized at all, which also brings energy savings.

\section{SENSOR NODE's OPERATION \& PERFORMANCE}

The operation of the implemented system has been tested in four various scenarios. The purpose of this is to show the capabilities of the implemented sensor node's hardware and software under different operation modes. In the first three scenarios, the sensor node is set to transmit a message containing overhead, capacitor charge level and temperature value every second, if it has sufficient amount of energy. Even though the one-second interval may not be practical for measuring temperature with a passive sensor node, this period was used as a reference to illustrate the operation principle and performance of the developed system. In the fourth scenario, the interval between transmitted packets is increased to five seconds and the sensor node has control over the smart light.

First, the sensor node was placed next to a window in a typical office environment when the sun was shining and office lights were on. The intensity of light coming from the sun is so strong that, not surprisingly, the sensor node was able to gather enough energy to compensate for its consumption (of about $168.2 \mu \mathrm{W}$ mean for 1 second reports) and had its supply voltage decently stable, as can be seen from Fig. 3 .

Fig. 4 shows the SoC's supply voltage level curve measured when the office lights were on, but curtains blocked the sun light. The sensor node ran out of energy after transmitting around 55 messages in average and then switched to a sleep mode. Once the energy in capacitor has been replenished, the sensor node switched back to active mode. The SoC leaves the sleep mode and samples the voltage level every five seconds, which explains, e.g., why it seems that the sensor node enters sleep mode at different voltage levels.

In the third scenario, the office lights are turned off, which makes the office very dim but not pitch black. Fig. 5 shows that voltage drops more rapidly than in Fig. 4 as expected. The sensor node can transmit around 30 broadcast packets with fully charged energy buffer before it exceeds a set threshold and enters sleep node. The average power consumption while transmitting and in sleep mode are $24 \mathrm{~mW}$ and $1.2 \mu \mathrm{W}$, respectively (see Figs. $6 \& 7$ ). If to consider a typical office where lights are on between 8 am and 4 pm, during the offoffice hours with no lights available the designed sensor node could transmit approximately twice per hour. During the weekend, this period may need to be increased, if the node is located far away from the windows.

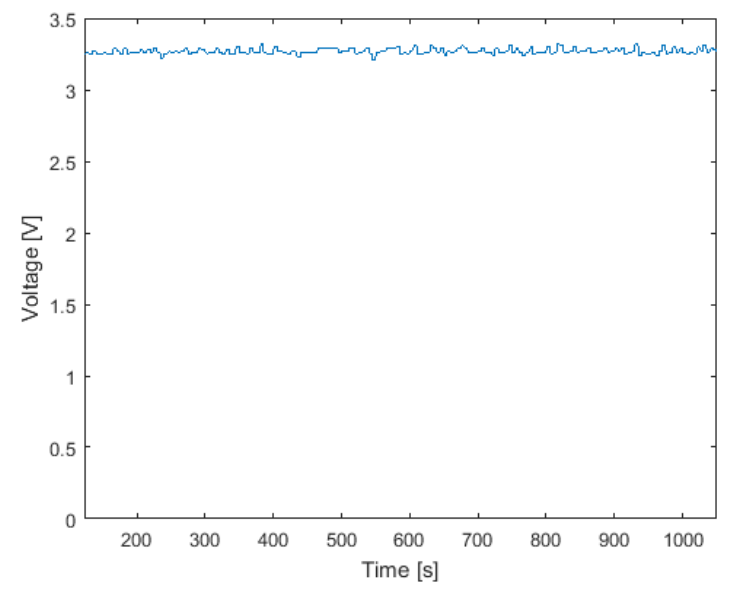

Fig. 3. Measured voltage with sensor node's ADC when photovoltaic panel is under sunlight.

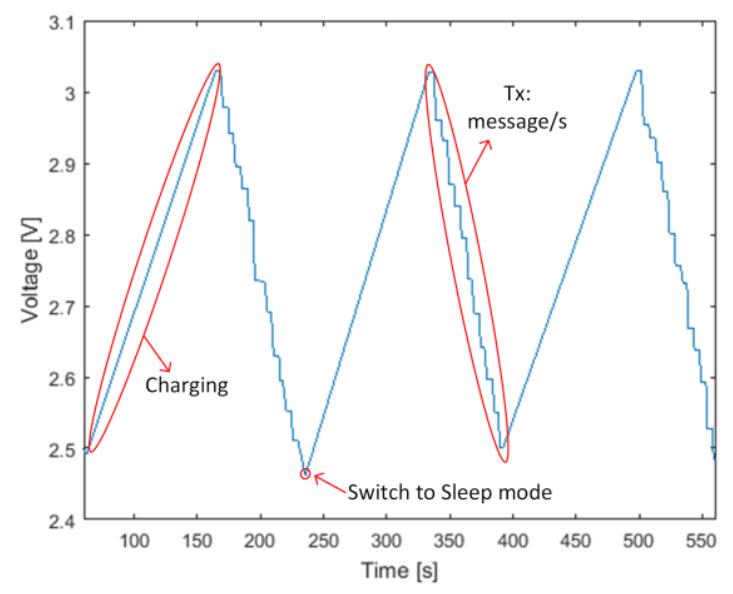

Fig. 4. Voltage level with typical office lighting.

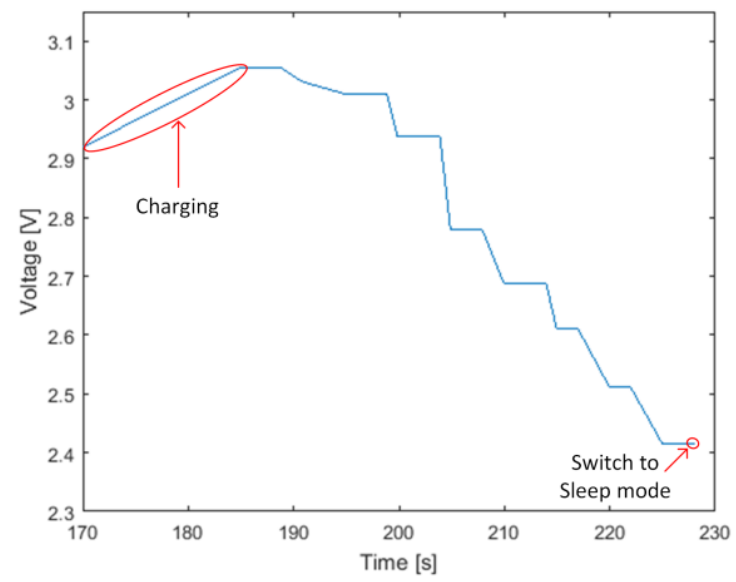

Fig. 5. Voltage level in a dim office while sensor node is transmitting at a rate of one message per second. 


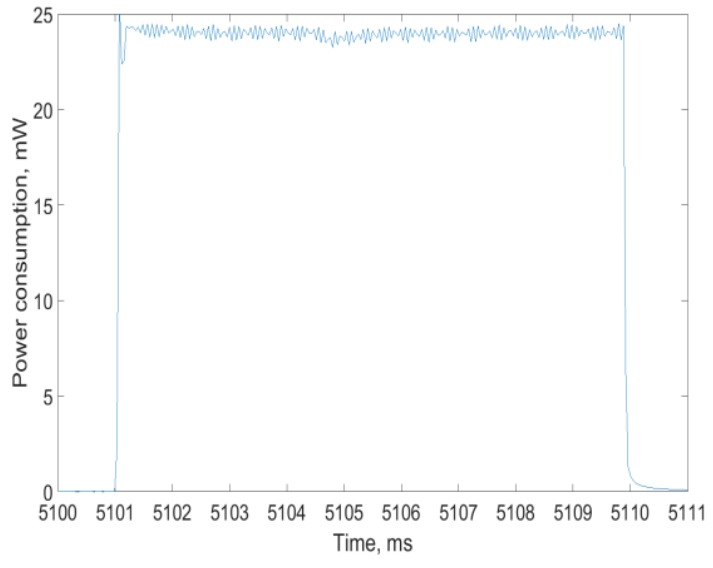

Fig. 6. Modular sensor node's power consumption in transmit mode.

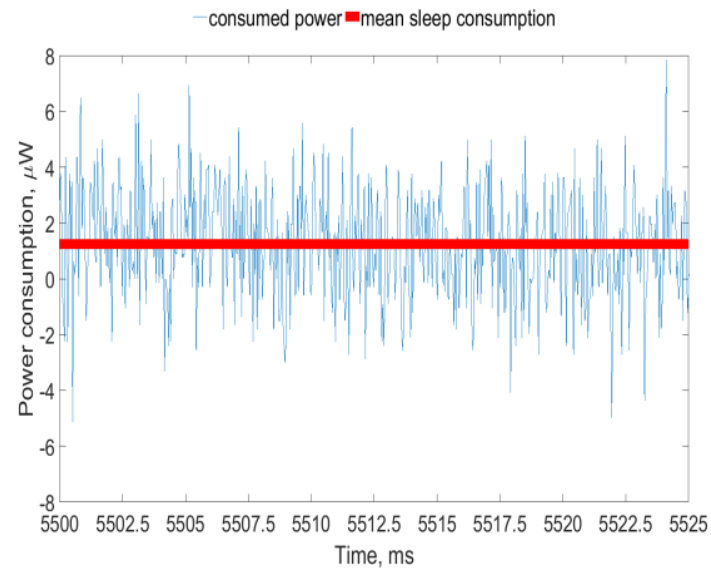

Fig. 7. Modular sensor node's power consumption in sleep mode.

In Fig. 8 it is shown how the voltage level changes when the sensor node has control over the smart light, which was the only light source in the environment. After the voltage level goes below the threshold (set to $2.9 \mathrm{~V}$ ), the sensor node transmits a request to the smart lamp to turn on. After the voltage level exceeds $3.2 \mathrm{~V}$, the sensor node transmits a request to turn off the light. Without communication or light blockage, the system can operate until component malfunction.

Note that during all the measurements made the reporting period of the sensor node stayed constant. The adjustment of the reporting period accounting for the available energy, current and expected energy income and the requirements of the applications can improve the efficiency of the proposed system further, reducing the number of light activation requests coming from the sensors. In addition, these mechanisms can be useful to handle the temporal blockage of the photovoltaic panels (e.g., by human body) or mitigate the loss of BLE requests due to interferences.

The proposed solution is qualitatively compared against the solutions featuring more conventional power supply options in

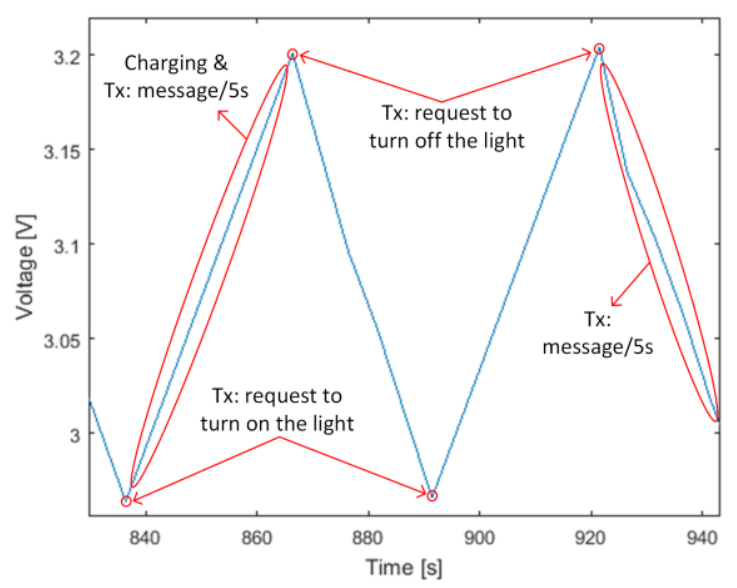

Fig. 8. The sensor node's voltage level when it is being charged at request.

Table I. As can be seen, the proposed solution has high starting costs since it requires investments in infrastructure, which should be made capable of receiving the commands from the sensor node. Nonetheless, e.g., the light fixtures of today are often equipped with means enabling them to communicate wireless and for them the added costs are void.

TABLE I. COMPARISON BETWEEN DIFFERENT OPTIONS FOR POWERING SENSOR NODES

\begin{tabular}{|l|c|c|c|c|}
\hline & Low & Low & Low & High \\
\hline $\begin{array}{l}\text { Infrastructure } \\
\text { cost }\end{array}$ & Quality of & High \\
service & High & Low & High \\
\hline Maintanance & $\begin{array}{c}\text { Battery } \\
\text { change } \\
\text { every } 1-10 \\
\text { years }\end{array}$ & $\begin{array}{c}\text { Battery } \\
\text { change } \\
\text { every } 1-10 \\
\text { years }\end{array}$ & Free & Free \\
\hline
\end{tabular}

\section{CONCLUSIONS}

Wireless sensor nodes, which are powered exclusively from energy harvesting, have not yet made a commercial breakthrough, even though energy harvesting has received a lot of attention from the academia. One of the fundamental reasons for this is the fact that for many potential applications the suitable energy sources are either not available or they are available only part of the time. In this paper, we addressed this problem by introducing a control loop between an energy harvesting sensor node and a nearly located controllable energy source. Using the proposed control loop, the sensor node can request an energy source, such as, e.g., a smart light, to turn on thus enabling the node to replenish its energy. By using the proposed method, the network can guarantee certain quality-ofservice for an application. 
To prove the feasibility of the proposed approach, a real life demonstrator composes of a photovoltaic energy harvesting enabled sensor node and a commercial smart light system, with control and reporting done via the BLE technology, has been implemented. The results of conducted measurements persuasively show the feasibility of the proposed concept in general and of the designed solutions in particular.

As future work, we plan to develop a passive sensor node with long-range communication capabilities using some LPWAN radio technology such as LoRaWAN or Sigfox. Data rates with these technologies are much lower than with BLE, which introduces challenges to the power harvesting and storage circuitry. Also we plan to investigate and improve the scalability and security aspects of the developed solution to enable its large scale deployment.

\section{REFERENCES}

[1] ABI research, "More than 30 billion devices will wirelessly connect to the internet of everything in 2020", 2013. [Online] https://www.abiresearch.com/press/more-than-30-billion-devices-willwirelessly-conne/

[2] Cisco. The internet of things - how the next evolution of the internet is changing everything. White Paper, 1-11, 2011. [Online] http://www.cisco.com/c/dam/en_us/about/ac79/docs/innov/IoT_IBSG_0 411FINAL.pdf

[3] J. F. Peters, M. Baumann, B. Zimmermann, J. Braun, M. Weil, "The environmental impact of Li-Ion batteries and the role of key parameters - A review", Elsevier Journal of Renewable and Sustainable Energy Reviews, vol. 67, pp. $491-506,2017$.

[4] National Renewable Energy Laboratory, "Best research-cell efficiencies", Technical report., 19.2.2017, [Online] https://www.nrel.gov/pv/assets/images/efficiency-chart.png.

[5] T. Nyländen, J. Boutellier, K. Nikunen, J. Hannuksela, and O. Silven, "Low-power reconfigurable miniature sensor nodes for condition monitoring," International Journal of Parallel Programming, pp. 1-21, 2014. [Online] http://dx.doi.org/10.1007/s10766-013-0302-5

[6] S. Sudevalayan and P. Kulkarni, "Energy Harvesting Sensor Nodes: Survey and Implications", IEEE Communications Surveys \& Tutorials, vol. 13, no. 3, pp. 443-461, 2011.
[7] M.-L. Ku, W. Li, Y. Chen, and K. J. R. Liu, "Advances in Energy Harvesting Communications: Past, Present, and Future Challenges", IEEE Communications Surveys \& Tutorials, vol. 18, no. 2, pp. 13841412,2016

[8] K. Z. Panatic, et al., "Energy Harvesting in Wireless Sensor Networks: A survey", Proc. IEEE International Symposium on Telecommunication Technologies, pp. 53-58, 2016.

[9] A.-R. El-Sayed, K. Tai, M. Biglarbegian, and S. Mahmud, "A Survey on Recent Energy Harvesting Mechanisms", Proc. IEEE Canadian Conference on Electrical and Computer Engineering, pp. 1-5, 2016.

[10] C. R. Valenta, and G. D. Durgin, "Harvesting Wireless Power: Survey of Energy-Harvester Conversion Efficiency in Far-Field, Wireless Power Transfer Systems", IEEE Microwave Magazine, vol. 15, no. 4, pp. 108120, 2014.

[11] X. Lu, P. Wang, D. Niyato, D. I. Kim, and Z. Han, "Wireless Charging Technologies: Fundamentals, Standards, and Network Applications", IEEE Communication Surveys \& Tutorials, vol. 18, no. 2, pp. 1413$1452,2016$.

[12] T. Soyata, L. Copeland, and W. Heinzelman, "RF Energy Harvesting for Embedded Systems: A Survey of Tradeoffs and Methodology", IEEE Circuits and Systems Magazine, vol. 16, no. 1, pp. 22-57, 2016.

[13] P. Ramezani, and M. R. Pakravan, "Overview of MAC Protocols for Energy Harvesting Wireless Sensor Networks", Proc. IEEE Personal, Indoor, and Mobile Radio Communications, pp. 2032-2037, 2015.

[14] R. Joshi, and J. Zhu, "Ambient energy harvester design for a wireless sensor network", Proc. IEEE International Conference on Electro/Information Technology, pp. 246-250, 2015.

[15] J. Janhunen, K. Mikhaylov, and J. Petäjäjärvi, "Experimental RF-signal based wireless energy transmission", Proc. European Conference on Networks and Communications, pp. 1-6.

[16] R. Kanan, and O. Elhassan, "Batteryless radio system for hospital application", Proc. SAI Computing Conference, pp. 939-945, 2016.

[17] Cypress, "CYALKIT-E03 Solar-Powered BLE Sensor 5 Pack”. [Online] http://www.cypress.com/documentation/development-kitsboards/cyalkite03-solar-powered-ble-sensor-5-pack

[18] K. Mikhaylov and J. Petäjäjärvi, "Design and Implementation of the Plug\&Play enabled Flexible Modular Wireless Sensor and Actuator Network Platform", Asian Journal of Control, vol. 19, no 5, pp. 1-21, 2017. 